\title{
A large and aggressive fibromatosis in the axilla: a rare case report and review of the literature
}

This article was published in the following Dove Press journal: OncoTargets and Therapy

\author{
Mingyue Duan' \\ Hua Xing' \\ Keren Wang' \\ Chunbo $\mathrm{Niu}^{2}$ \\ Chengwei Jiang ${ }^{2}$ \\ Lijuan Zhang' \\ Shereen Ezzat ${ }^{3}$ \\ Le Zhang'
}

'Department of Breast Surgery, China-Japan Union Hospital, Jilin University, Changchun, Jilin, People's Republic of China; ${ }^{2}$ Department of Pathology, China-Japan Union Hospital, Jilin University, Changchun, Jilin, People's Republic of China; ${ }^{3}$ Ontario Cancer Institute and The Endocrine Oncology Site Group, Princess Margaret Hospital, University Health Network, Toronto, ON, Canada
Correspondence: Le Zhang Department of Breast Surgery, China-Japan Union Hospital, Jilin University, 126 Xiantai Street, Jingkai District, Changchun, Jilin I30033, People's Republic of China

Tel +8643184995495

Fax +8643184995495

Email lezhangmed@163.com

\begin{abstract}
Aggressive fibromatosis (AF) is a rare benign tumor, which occurs in the deep part of bone and muscle fibrous tissue. Clinical and pathological features can be challenging for definitive diagnosis. Here, we report a rare case of a large AF in the axilla. Interestingly, 18 F-fluorodeoxyglucose-positron emission tomography/computed tomography showed significant increase in standard uptake value. Surgical resection yielded a spindle cell tumor likely of fibromatosis origin which was positive for $\beta$-catenin expression.
\end{abstract}

Keywords: aggressive fibromatosis, desmoid-type fibromatosis, axilla, 18 F-fluorodeoxyglucose, $\mathrm{PET} / \mathrm{CT}, \beta$-catenin

\section{Introduction}

Fibromatosis is a benign tumor caused by the proliferation of differentiated fibroblasts, and aggressive fibromatosis (AF) is a subtype of fibromatosis, also known as desmoid tumor or desmoid-type fibromatosis (DF), which is a monoclonal fibroblastic proliferative disease. ${ }^{1}$ The disease occurs frequently in the abdomen and extremities but very rarely in the axillae. While metastatic spread is typically not seen, the disease often presents as a local infiltrating mass with significant compressive features. Despite lack of negative impact on survival, recurrence rates can be high. We encountered a rare case of large AF in axilla where immunohistochemical studies showed strong $\beta$-catenin expression. Interestingly, 18 F-fluorodeoxyglucose (FDG)-positron emission tomography/computed tomography (PET/CT) imaging showed significant uptake similar to that observed in malignant tumors.

\section{Case report}

A 54-year-old female patient with no smoking or alcohol consumption history presented with a left axillary mass. She had undergone bilateral accessory breast resection 20 years earlier. This left axillary mass was first noted in 2012 but the patient declined treatment until December 2016. By then, the tumor had reached $10 \times 8 \mathrm{~cm}^{2}$ limiting left upper arm motor function (Figure 1). There was no pain or sensory complaints related to this large tumor.

Ultrasound examination revealed two regular hypoechoic masses $\left(5 \times 3 \mathrm{~mm}^{2}\right)$ in the left breast. Color doppler signal demonstrated absent blood flow. In addition, there was an irregular and inhomogeneous low echogenic mass in the subcutaneous soft tissue deep within the left axilla measuring $8 \mathrm{~cm}$ in longest diameter (Figure 2A) with irregular borders and scant blood flow signal. Plain and contrast-enhanced magnetic resonance imaging (MRI) examination of breast revealed that a nodular and oval acoustic shadow with clear and smooth border in the upper inner quadrant of the left 

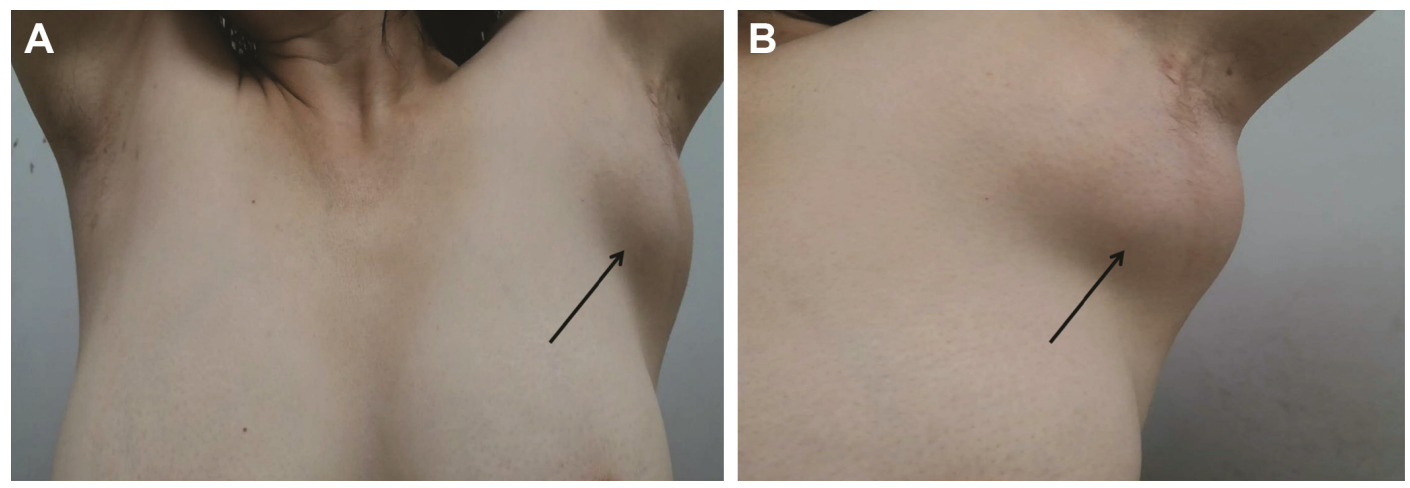

Figure I Preoperative axillary photo of patient.

Notes: Before surgery, the large mass on the left axillary was visibly prominent (A and $\mathbf{B})$. Arrows indicate the lesion of interest.

breast. In contrast, the mass within the left axilla revealed irregular borders and signal (Figure 2B). FDG-PET/CT showed that the saccharometabolism of the left axillary mass was inhomogeneous with increased standard uptake value (SUV) max value of 3.80. The lesion could not be easily separated from adjacent muscle including the musculi teres minor, the subscapalaris, and the scapula itself (Figure 3). A core needle biopsy demonstrated fibrous tissue with glassy degeneration and focal vascular hyperplasia (Figure 4). As these results precluded a definitive diagnosis, surgical excision was recommended and performed.
At the time of surgery, the mass was extremely hard with an incomplete capsule and could not be easily separated from surrounding tissues (Figure 5). Indeed, adjacent structures were deformed and displaced because of the tumor's mass effect which reached deep to the clavicle and invaded the surrounding tissues including: pectoralis major, brachial plexus, subscapalaris, latissimus dorsi, teres minor, and scapula. Intraoperative pathology result suggested a spindle cell tumor (Figure 6A) which was confirmed on permanent paraffin sections with infiltration into striated muscle (Figure 6B). Tumorous tissue was negative for $\mathrm{Bcl} 2$; and
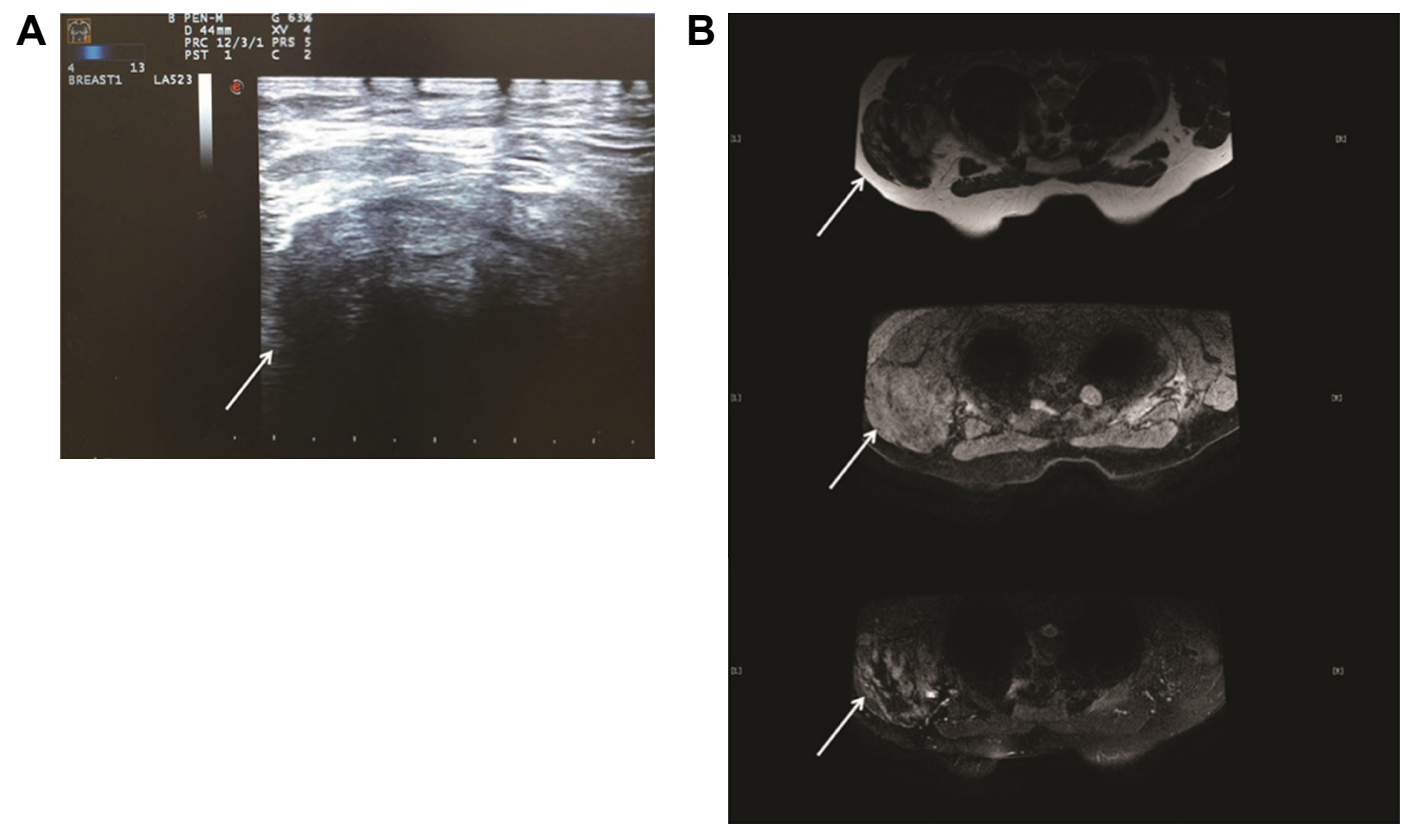

Figure 2 Imaging of ultrasound and MRI examination of breast.

Notes: (A) A low echo mass of $8 \times 5 \mathrm{~cm}^{2}$ can be seen in the left axilla by ultrasound examination. The boundary was unclear, and the shape was irregular. (B) The mass in the left axilla manifested as unclear boundary and unequal signal by MRI. TIWI and T2WI revealed mixed signal with uneven enhancement. Arrows indicate the lesion of interest.

Abbreviation: MRI, magnetic resonance imaging. 


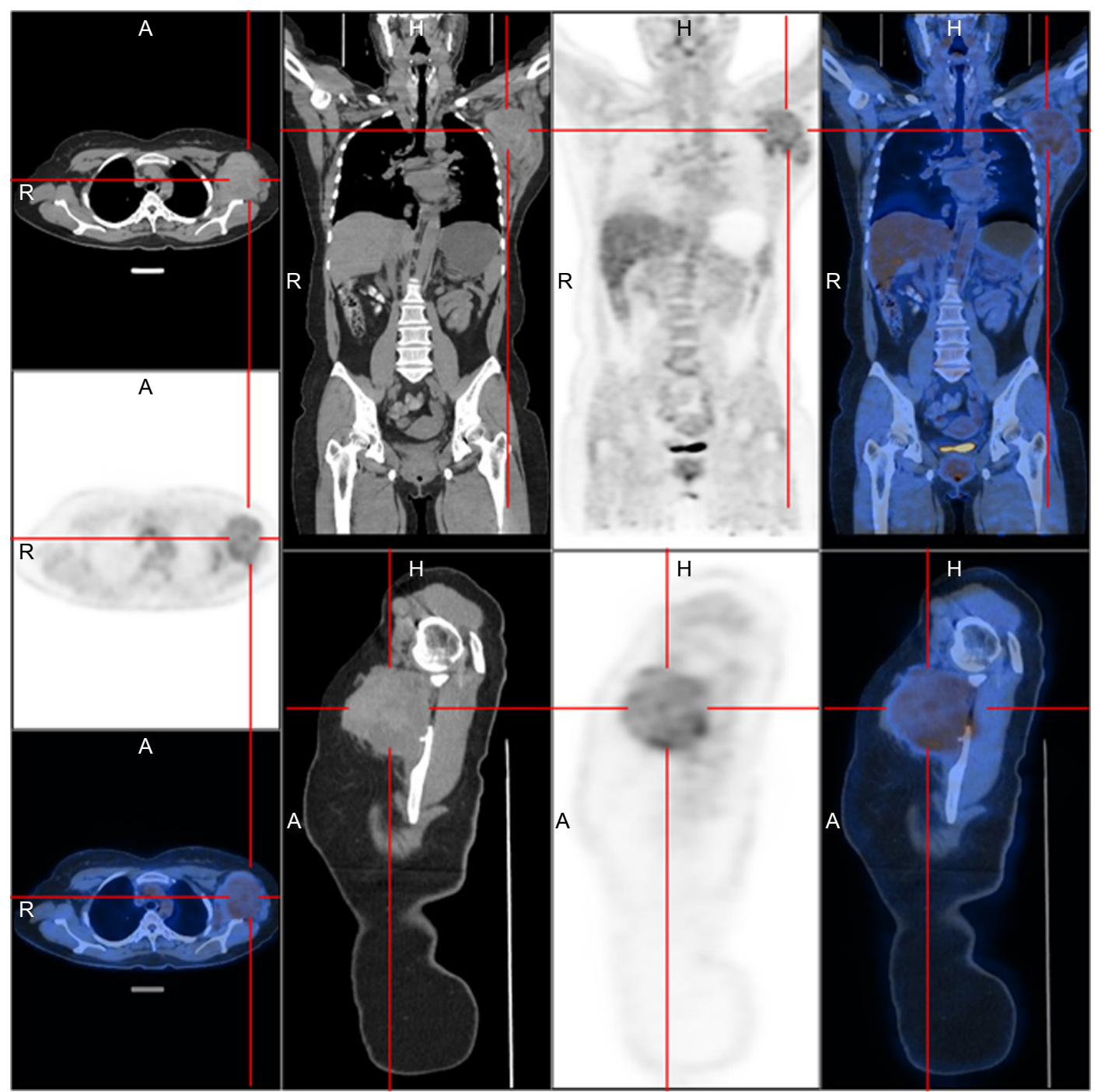

Figure 3 Imaging of 18 F-fluorodeoxyglucose (FDG) PET/CT.

Notes: The saccharometabolism of the left axillary mass was in homogeneously increased and the lesions were not clear with the boundary of musculi teres minor, subscapalaris and the scapula on the left. Left side vertical images (transverse section of the chest): The images from top to bottom are CT plain scan, PET plain scan, and the synthesis of PET and CT images. Upper right images (front projection of human body): The images from the second to the fourth are CT plain scan, PET plain scan, and the synthesis of PET and CT images. Lower right images (side projection of human body): The images from the second to the fourth are CT plain scan, PET plain scan, and the synthesis of PET and CT images.

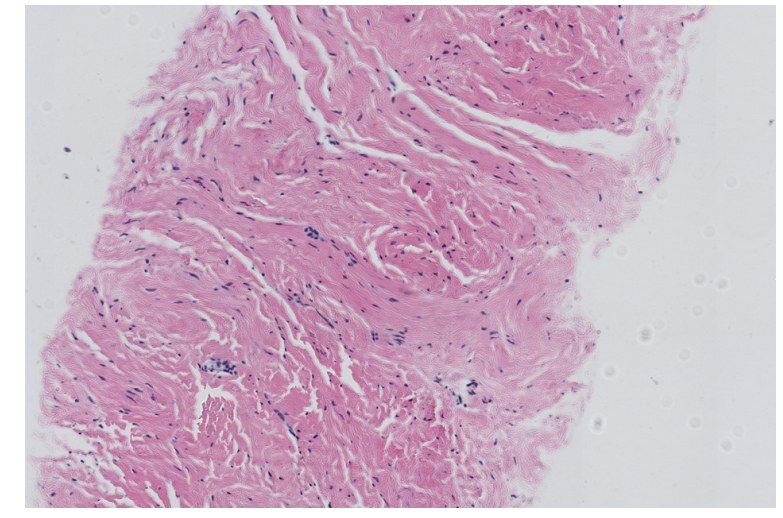

Figure 4 Image of core needle biopsy histologic diagnosis using hematoxylin and eosin staining (scale bar $=2,000 \mu \mathrm{m}$; original $\times 100$ ).

Note: Glassy degeneration and focal vascular hyperplasia can be seen in the fibrous tissue. positive for $\beta$-catenin, smooth muscle actin, vimentin, and CD34 (Figure 7). Based on these findings, the patient was diagnosed with a left axillary AF.

After surgery, we asked for self- or family history of fibromatosis which were denied. The patient recovered soon without adjuvant medical or radiation therapy. After more than 1 year of follow-up, the patient remains in good condition, without cutaneous or muscle AF recurrence.

\section{Discussion}

AF is a subtype of fibromatosis, which refers to fibrous tissue tumors that occur in bones and muscles. AF is divided into superficial and deep types wherein the latter is also called desmoid tumors or DF representing a monoclonal cell 

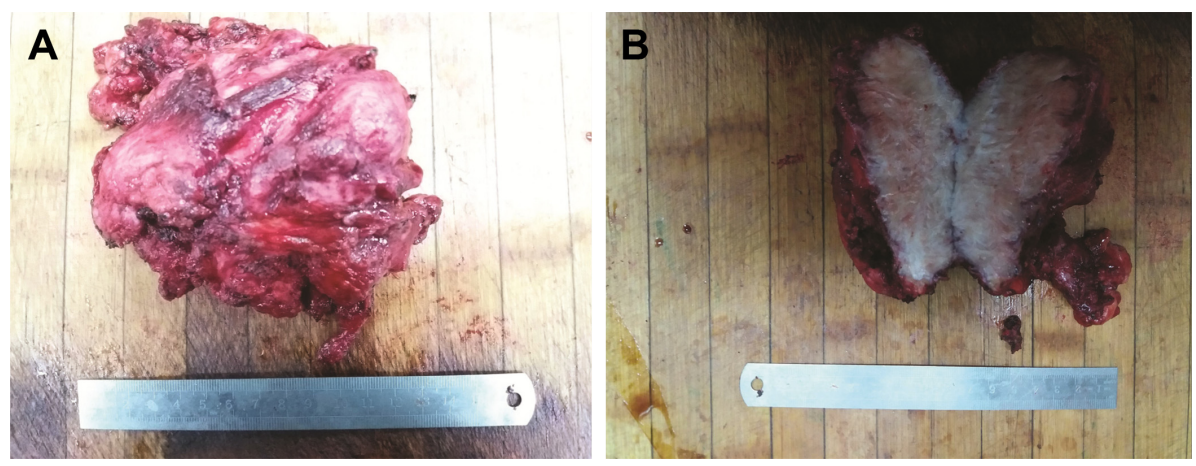

Figure 5 Intraoperative photo of the mass.

Notes: (A) The complete appearance of the tumor. (B) Tumor section image. The mass was large $\left(|2 \times| I \mathrm{~cm}^{2}\right)$, and the section of the mass was presented as fibrous tissue interleaving.

proliferative disease. ${ }^{1}$ DF is composed of spindle cells and collagen fibrils, which accounts for $0.03 \%$ of all tumors and about $3 \%$ of all soft tissue tumors. ${ }^{2}$ DF is most frequently encountered in the abdomen, shoulders and limbs, and rarely in the axillae. ${ }^{3,4}$ Spindle cell tumors can occur in epithelial or mesenchymal tissues. There is no obvious effect on the survival rate of patients. However, the location of the DF can result in significant morbidities. These tumors often invade and compress surrounding tissues ${ }^{5}$ with a high recurrence rate. ${ }^{2}$

Although there are some reports of AF, it is rare for them to develop in the axilla and reach to the deep side of the clavicle. The growth rate can be variable depending on the abundance of blood supply. In our case, we believe the rich axillary vasculature allowed the tumor to reach the $10.0 \times 8.0 \times 5.0 \mathrm{~cm}^{3}$ resulting in invasion of pectoralis major, latissimus dorsi, teres minor, scapula muscle, scapular nerve, and brachial plexus.

Unfortunately, the rarity of these tumors precludes clear diagnostic and treatment guidelines. At present, the first choice of management includes MRI and core needle biopsy examination. However, it is important to note that final diagnosis required permanent sections examination of surgically excised tissue. ${ }^{6}$ In particular, immunohistochemistry (IHC) detection of $\beta$-catenin expression can be used as one feature supportive of the diagnosis of AF. ${ }^{7-9}$ More recently, FDG $\mathrm{PET} / \mathrm{CT}$ has been widely applied for clinical staging. It should be emphasized, however, that the increased SUV values can be noted in both benign- and malignant-behaving tumors. ${ }^{10}$ Some researchers defined malignancy based on the 2.5 times of threshold of SUV max. ${ }^{11}$ In this regard, DF is similar to breast fibroadenomas where 18 F-FDG PET/CT thresholds cannot reliably distinguish benign from malignant diseases. ${ }^{10}$

Surgical treatment is the preferred approach to the management of patients with suspected AF. It has been reported that radical surgery is the most important treatment to avoid disease recurrence. ${ }^{2}$ Negative resection margins can ensure long-term remission. Nevertheless, the extent of surgical resection currently remains controversial. ${ }^{12}$ In fact, more recent reports have advocated active surveillance as a reasonable management strategy. At the other end of the spectrum, chemotherapy and radiation can be considered in some unresectable or incompletely resected tumors. ${ }^{13,14}$ Additional studies reported that the location of DF affected the patient's event-free survival (EFS) after the treatment. ${ }^{14}$ In particular, disease involving the abdominal wall, abdominal cavity,
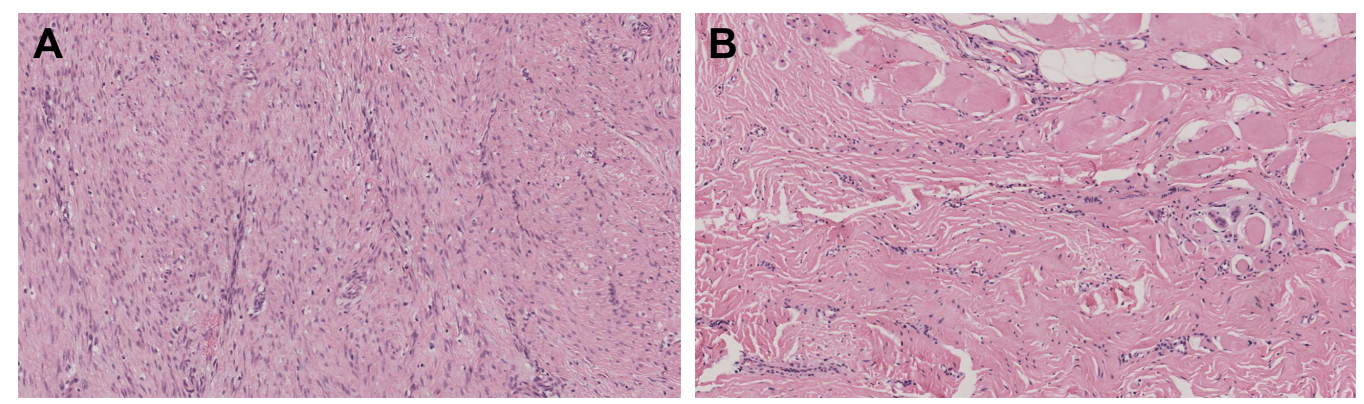

Figure 6 Image of paraffin pathology histologic diagnosis using hematoxylin and eosin staining (scale bar =2,000 $\mu \mathrm{m}$; original $\times 100$ ).

Notes: (A) An abundance of spindle cells is evident. (B) Tumor involvement in striated muscle is seen. 

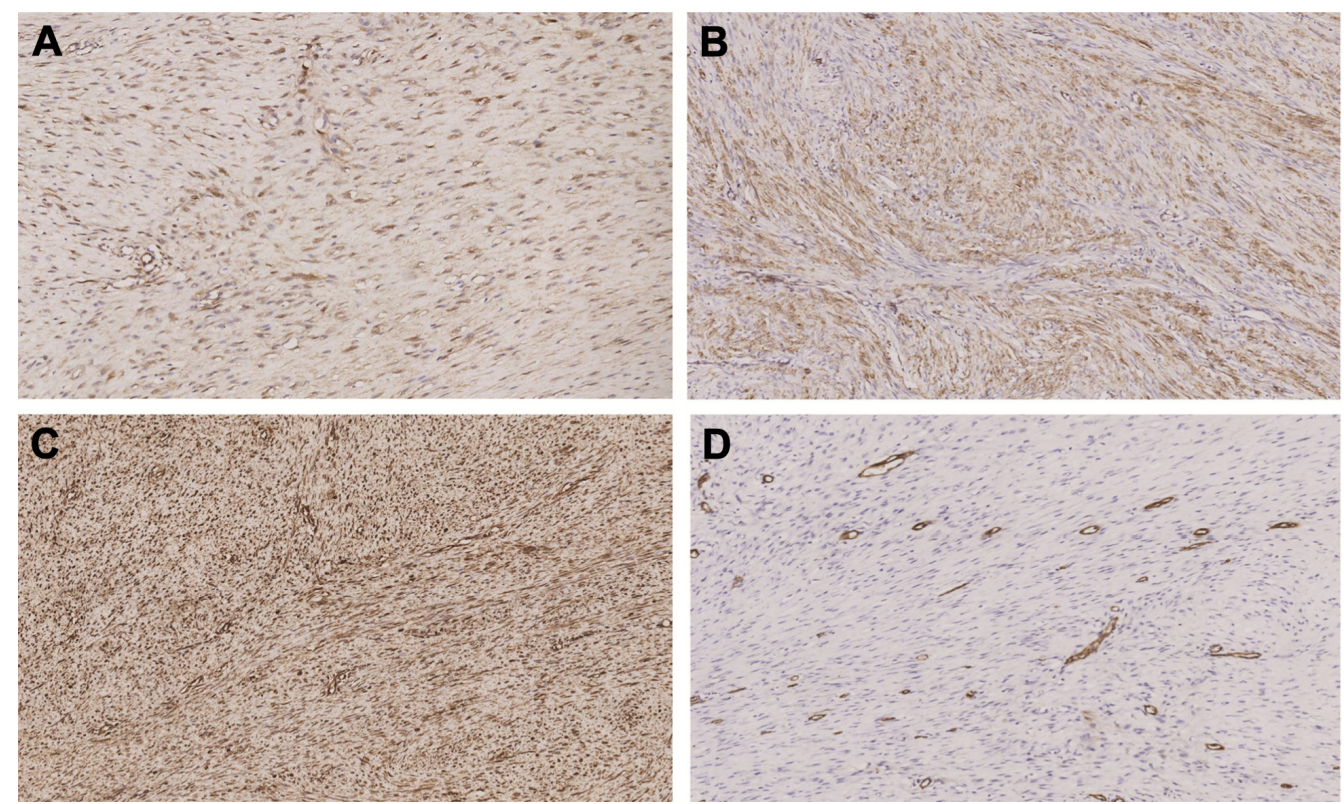

Figure 7 Expression of $\beta$-catenin, SMA, vimentin, and CD34 by IHC staining (scale bar $=2,000 \mu \mathrm{m}$; original $\times 100$ ).

Notes: (A) $\beta$-Catenin positive expression can be detected by IHC staining. (B) SMA positive expression can be detected by IHC staining. (C) Vimentin positive expression can be detected by $\mathrm{IHC}$ staining. (D) CD34 positive expression can be detected by $\mathrm{IHC}$ staining.

Abbreviations: IHC, immunohistochemistry; SMA, smooth muscle actin.

breast, digestive viscera, and lower limbs appear to behave favorably. For these anatomical sites, the EFS was similar between surgical treatment and active surveillance. By comparison, unfavorable locations show significantly improved EFS for surgical treatment. ${ }^{14}$ However, in the case presented here, the axillary location is considered to be one of the "unfavorable sites" due to complex and critical neural and vascular supplies. Moreover, the patient's left upper arm was functionally affected by the extra-large size of the tumor providing more rationale for surgical intervention.

As indicated earlier, radiotherapy can also be an effective form of treatment for AF, especially for DF in the limbs. ${ }^{15}$ Janssen et al found that with analysis of 1,295 cases, the risk of local recurrence following surgical resection was two times higher in the presence of positive margins. In such cases, postoperative adjuvant radiotherapy seems to reduce the risk of recurrence. ${ }^{13}$ Although radiotherapy can lead to growth arrest, it can also be associated with side effects such as pain, limb edema, and skin toxicity. ${ }^{16}$

In recent years chemotherapy and biologically targeted therapies have also received attention. ${ }^{17}$ Park et al used combination chemotherapy of methotrexate and vinblastine for unresectable AF. ${ }^{18}$ The results showed that half of patients presenting with pain experienced tumor size reduction. The most common adverse reaction was the anticipated liver enzyme elevation in nearly $80 \%$ of patients. Low-dose methotrexate and vincristine combination therapy was effective and well-tolerated..$^{18}$ It has been reported that non-steroidal anti-inflammatory drugs (NSAIDs), hormones and tyrosine kinase inhibitors (TKIs) were all feasible options for longterm control of AF. However, the mechanism underlying the efficacy of TKI remains unclear. ${ }^{18}$ For NSAIDs, Pruksakorn et al completed successful steroid injections in eight patients with recurrent AF. ${ }^{19}$

\section{Conclusion}

$\mathrm{AF}$ is a benign tumor with local infiltration and growth potential. In particular, $\beta$-catenin can be used as tissue biomarker for diagnosis and prognostic value. FDG PET/CT can also provide additional information to assist with surgical planning. Radiotherapy may have some beneficial effect by reducing the risk of recurrence in cases of incomplete resection. In contrast, the role of steroids, NSAIDs, and targeted therapies remains to be determined.

\section{Acknowledgments}

This study received approval from the ethics committee of The China Japan Union Hospital of Jilin University. The patient and family members also provided written informed consent to publish the case report details including photography.

\section{Disclosure}

The authors have no conflicts of interest in this work. 


\section{References}

1. Foa R, Rizzo S, Petrella F, De Maria F, Bellomi M. Recurrent aggressive fibromatosis of the chest wall. Ecancermedicalscience. 2014;8:464.

2. Hajjar WM, AlShehri AF, Alessa MA, Al-Nassar SA. Late presentation of aggressive fibromatosis involving head, neck and chest wall. $J$ Coll Physicians Surg Pak. 2017;27(10):654-656.

3. Lee SH, Lee HK, Song JS, Jeong HS. Chest wall fibromatosis in the axilla. Arch Plast Surg. 2012;39(2):175-177.

4. Martinez Trufero J, Pajares Bernad I, Torres Ramon I, Hernando Cubero J, Pazo Cid R. Desmoid-type fibromatosis: who, when, and how to treat. Curr Treat Options Oncol. 2017;18(5):29.

5. Smith AJ, Lewis JJ, Merchant NB, Leung DH, Woodruff JM, Brennan MF. Surgical management of intra-abdominal desmoid tumours. Br J Surg. 2000;87(5):608-613.

6. Zhang YJ, Gao YS. [Clinical features and surgical treatment of chest aggressive fibromatosis]. Zhonghua Zhong Liu Za Zhi. 2016;38(3): 232-235. Chinese.

7. Yang S, Wang X, Jiang H, Wang Y, Li Z, Lu H. Effective treatment of aggressive fibromatosis with celecoxib guided by genetic testing. Cancer Biol Ther. 2017;18(10):757-760.

8. Skubitz KM. Biology and treatment of aggressive fibromatosis or desmoid tumor. Mayo Clin Proc. 2017;92(6):947-964.

9. Sakai T, Nishida Y, Hamada S, et al. Immunohistochemical staining with non-phospho beta-catenin as a diagnostic and prognostic tool of COX-2 inhibitor therapy for patients with extra-peritoneal desmoid-type fibromatosis. Diagn Pathol. 2017;12(1):66.

10. Hofman MS, Hicks RJ. How we read oncologic FDG PET/CT. Cancer Imaging. 2016;16(1):35.
11. Hain SF, Curran KM, Beggs AD, Fogelman I, O’Doherty MJ, Maisey MN. FDG-PET as a "metabolic biopsy" tool in thoracic lesions with indeterminate biopsy. Eur J Nucl Med. 2001;28(9):1336-1340.

12. Harati K, Jaenisch A, Behr B, et al. Effect of surgical margins on prognosis in aggressive fibromatosis: a single-institutional analysis of 90 patients. Oncol Lett. 2017;14(5):5129-5134.

13. Janssen ML, van Broekhoven DL, Cates JM, et al. Meta-analysis of the influence of surgical margin and adjuvant radiotherapy on local recurrence after resection of sporadic desmoid-type fibromatosis. $\mathrm{BrJ}$ Surg. 2017;104(4):347-357.

14. Penel N, Le Cesne A, Bonvalot S, et al. Surgical versus non-surgical approach in primary desmoid-type fibromatosis patients: a nationwide prospective cohort from the French Sarcoma Group. Eur J Cancer. 2017; 83:125-131.

15. Zlotecki RA, Scarborough MT, Morris CG, et al. External beam radiotherapy for primary and adjuvant management of aggressive fibromatosis. Int J Radiat Oncol Biol Phys. 2002;54(1):177-181.

16. Scheer L, Lodi M, Moliere S, Kurtz JE, Mathelin C. Medical treatment of mammary desmoid-type fibromatosis: which benefit? World J Surg Oncol. 2017;15(1):86.

17. Palassini E, Frezza AM, Mariani L, et al. Long-term efficacy of methotrexate plus vinblastine/vnorelbine in a large series of patients affected by desmoid-type fibromatosis. Cancer J. 2017;23(2):86-91.

18. Park KH, Choi YJ, Kim KW, et al. Combination chemotherapy with methotrexate and vinblastine for surgically unresectable, aggressive fibromatosis. Jpn J Clin Oncol. 2016;46(9):845-849.

19. Pruksakorn D, Lorsomradee S, Phanphaisarn A, et al. Safety and efficacy of intralesional steroid injection for aggressive fibromatosis. World $J$ Surg Oncol. 2017;15(1):195.
OncoTargets and Therapy

\section{Publish your work in this journal}

OncoTargets and Therapy is an international, peer-reviewed, open access journal focusing on the pathological basis of all cancers, potential targets for therapy and treatment protocols employed to improve the management of cancer patients. The journal also focuses on the impact of management programs and new therapeutic agents and protocols on

\section{Dovepress}

patient perspectives such as quality of life, adherence and satisfaction. The manuscript management system is completely online and includes a very quick and fair peer-review system, which is all easy to use. Visit http://www.dovepress.com/testimonials.php to read real quotes from published authors. 